\title{
Postgraduate nurses' insights into the nursing leadership role. Do they intuitively link the role to patient safety?
}

\author{
Joyce Hendricks , Vicki Cope, Gwendolyn Baum \\ School of Nursing and Midwifery, Edith Cowan University, Joondalup, Australia
}

Received: March 30, 2015

DOI: $10.5430 /$ jnep.v5n9p72

\author{
Accepted: May 17, 2015 \\ Online Published: June 23, 2015 \\ URL: http://dx.doi.org/10.5430/jnep.v5n9p72
}

\begin{abstract}
Nursing leaders are compelled to ensure a safety and quality agenda in the acute care environment as patient outcomes are linked to nursing care. Good nursing leadership where focus is directed to accountability and responsibility for clinical outcomes and patient safety, results in reduced adverse events and patient mortality. Integral to the future of high-quality effective clinical care with an absence of errors is the training of the next generation of nursing leaders. In this discussion paper, the extent to which future nurse leaders intuitively acknowledge patient safety as part of their leadership role was examined amongst a cohort of postgraduate nursing students. A content analysis to search for quality and safety terminology was conducted on 146 essay responses to a question about the nurse leader role in today's healthcare environment. The results indicated minimal acknowledgement of patient safety as an intuitive consideration in the nursing leadership role. Recommendations are discussed for developing postgraduate nursing educational curricula with patient safety strategies as a central component to the practice of leadership.
\end{abstract}

Key Words: Leadership, Patient safety, Quality improvement, Nursing education, Postgraduate nursing students

\section{INTRODUCTION}

Despite substantial research and resources devoted to understanding patient safety, preventable medical errors are on the rise in developed nations and can affect up to $10 \%$ of hospitalised patients. ${ }^{[1]}$ This is evidenced by the alarming number of published reports into hospital safety and system errors within the last twenty years ${ }^{[2,3]}$ should correlate with a safety and quality agenda that would be foremost in healthcare workers' consciousness. This article grew from this premise, that nurses (in this case postgraduate nursing students) would be congruent with this topic and realise how their role in nursing leadership can make an impact. What is known is that patient quality and safety goals may be hindered without the ongoing involvement of nurses. Nurse leaders have a role in ensuring a safety and quality agenda ${ }^{[1,4]}$ especially in the acute care environment where patient outcomes are linked to nursing care. Hours of nursing care provided and skill mix are pivotal to patient safety and reducing adverse events, and the role of the leader is central to creating a safe environment for the patient in the hospital. ${ }^{[5,6]}$

The literature clearly demonstrates a correlation between good leadership and patient safety outcomes. ${ }^{[7]}$ More recently clinical governance frameworks have articulated the accountability and responsibility for clinical outcomes and patient safety to all levels of care providers in the organ-

\footnotetext{
*Correspondence: Joyce Hendricks; Email: j.hendricks@ecu-edu.au; Address: School of Nursing and Midwifery, Edith Cowan University, Joondalup, Australia.
} 
isational hierarchy. This has meant that nurses who have historically deferred to a higher level on issues concerning clinical care and safety are all now accountable and responsible for decisions and outcomes concerning nursing care, safety and quality within the scope of their practice. ${ }^{[8]}$

Given the evidence in the literature, a fundamental appreciation of what nurses at the coalface understand as their role as leaders with responsibility for safety issues, required elaboration. The researchers wanted to verify if our postgraduate nursing students made the link between leadership and safety in their own practice. Therefore, postgraduate student nurses understanding of leadership was explored and examined through content analysis to ascertain the relationship between the perceptions of the leadership role and its impact on patient safety. During the content analysis, the researchers looked specifically for words related to safety and quality, as the researchers felt these words, terms and phrases would, and should, have evidenced intuitive thinking establishing a link between leadership and safety.

Benner's ${ }^{[9]}$ novice to expert research suggested that a nurse's development of clinical reasoning and critical thinking skills are dependent on continual learning, and one component of critical thinking is intuition which influences the nurse to provide safe, high-quality care. Further, intuitive knowledge as a basis for arriving at clinical judgements is useful when coupled with appropriate evidence for developing new ways to consider nursing situations. In this discussion paper, intuitive knowledge was believed to underpin and provide the link between leadership, patient safety, quality and the considerations related to managing patient risk.

\subsection{Search strategy}

A literature search was performed to identify the body of research related to safety and nursing published from 2000 to 2012 using the CINAHL and MEDLINE databases. The following keywords were used using Boolean commands 'and', and 'or' with 'nursing' and the related words, 'quality', 'safety', 'patient risk', 'adverse event', 'medical error', and 'quality improvement'. Limitations imposed on the search included relevance to the topic with a preference for peer reviewed original research works published in English. Over 36,000 citations were viewed with 45 articles providing the basis for subsequent investigation of the terms identified. Based on the review of the literature, an understanding was developed which informed the link between leadership and safety as a construct that nurses should use to guide practice.

\subsection{Background}

Reports such as the Institute of Medicine Keeping Patients Safe: Transforming the Work Environment of Nurses ${ }^{[10]}$ ac-

Published by Sciedu Press knowledge the role of front-line nurses in facilitating patient care and thus their safety. An American study by Cook, Hoas, Guttmannova, and Joyner ${ }^{[11]}$ found that medical staff, administrators and nurses believed that the quality of care for patients was mainly a nursing responsibility, with ninetysix per cent of nurses and over ninety per cent of medical and allied health assigning primary responsibility for patient safety to nursing. Only twenty-two per cent of those surveyed believed that patient safety was a shared responsibility. It therefore begs the question, if nurses in America believe the safety of the patient lies largely within their domain, do nurses at the grassroots in Western Australia share this view given that patient safety is embedded in their job descriptions and contained in the Australian Nursing and Midwifery Competencies (ANMC) ${ }^{[12]}$ that govern their practice?

The recent highlighted flaws within the Australian healthcare system and overseas has evidenced concerns related to patient safety and clinical risk. ${ }^{[13-15]}$ A number of these reports and enquires have identified major system inadequacies as the main reason for the failure of safety mechanisms within healthcare. However, Gregory, Guse, Dick and Russell ${ }^{[16]}$ asserted that the focus of patient safety should now move from a systems approach to an individual accountability and responsibility framework commencing within undergraduate education and continuing throughout a nurse's professional journey. Chernot and Daniel ${ }^{[17]}$ contended that this builds a safety vision and a sense of personal responsibility for ensuring that systematic planning for addressing problems is part of professional practice and a holistic shift in building an emphasis on patient safety, merging the role of an individual with the organisational system, in keeping within the premises of "real" clinical governance. ${ }^{[18]}$ Therefore, for real governance to occur, nurses on the front line need to embrace the mantle of patient safety. ${ }^{[19]}$

It is critical that nurses in the practice setting maintain a quality and safety focus because the consequence of not doing so is detrimental. Preventable medical errors in hospitals are attributed to loss of human lives, additional hospital expense, and loss of trust in the healthcare system. ${ }^{[5]}$ At an operational level nurses are expected to collaborate with other disciplines, work within a set of standards, monitor patient status, advocate on behalf of patients and delegate to healthcare workers in a variety of settings. In order to undertake these roles efficiently every registered nurse (RN) must be prepared to take the lead to act autonomously, make decisions at the point of service, and develop a professional vision that fits, not only organisational goals, but with professional aspirations. ${ }^{[20]}$

Patient safety as a core component of a nurse leader's role is often overlooked. This may be because the traditional view of 
leadership maintains the traditional hierarchical structure of the organisation, perpetuating the view that patient safety is someone else's role, despite documentation that purports that all health professionals, including nurses, at the grassroots have a responsibility for safety. Higgs and Rowland ${ }^{[21]}$ (p. 1) coined the phrase "stuckness" whereby nursing leaders fail to recognise systemic issues concerning patient safety and quality and either consciously or unconsciously reinforce the systemic patterns that cause the organisation to remain "stuck". This "stuckness" prevents necessary change concerning the responsibility for patient safety and accountability at the bedside and impairs the implementation of change strategies to enhance patient safety. Conversely, some would argue that the hierarchical structure has been flattened and that transformational models of leadership empower followers, in this case nurses, to share in the responsibility of safety to promote positive change in individual nurse's responsibility and accountability concerning patient safety, avoided the "traps" incumbent in hierarchical hospital management and the paralysis concerning implementing a safety agenda. ${ }^{[21-23]}$

Chernot and Daniel ${ }^{[17]}$ and Steven, Magnusson, Smith and Pearson ${ }^{[24]}$ evidenced that nurses are the most likely health professional to recognise, interrupt and correct clinical errors. There is a paucity of evidence however, that the majority of nurses are prepared to assume the leadership role required to examine patient safety issues as an integral part of their practice, even though broad outcomes have been incorporated into nursing competency and accreditation statements for several years. ${ }^{[25]}$ For example, the ANMC ${ }^{[12]}$ competency statement numbered 1.3 for the registered nurse requires that an RN: "Recognises and responds appropriately to unsafe or unprofessional practice... and identifies appropriate action to be taken in specified circumstances." In the United Kingdom, the Code for Nurses ${ }^{[26]}$ states that "as a professional, you are personally accountable for actions and omissions in your practice and must always be able to justify your decisions". The American Nurses Association (ANA) ${ }^{[27]}$ believe that: "The nurse's primary commitment is to the health, well-being, and safety of the patient."

In order to practice within these competencies and codes, registered nurses must be equipped with the critical thinking skills to recognise safety as part of their role. If emerging nurse leaders are not encouraged to actively think for themselves from a safety perspective, they may be hindered in the development of these skills. Wakefield et al. ${ }^{[28]}$ identified the potential for nursing curricula, at under and postgraduate levels to enable nurses to examine patient safety issues. Vaismoradi, Salami and Marck ${ }^{[29]}$ (p. 434) also found that "nursing education curriculum designers need to go beyond theoretical concepts of patient safety education and devise strategies to increase the application of safety knowledge and competencies in nursing practice". Steven, Magnusson, Smith, and Pearson ${ }^{[24]}$ stress that a safety education curriculum remains a "hidden curriculum or practice culture" (p. $1)$.

\section{METHOD}

\subsection{Aim}

This study aimed to determine the extent to which potential nurse leaders enrolled in a Master of Nursing program intuitively acknowledge patient risk and safety as part of their leadership role.

\subsection{Participants}

One hundred and forty six $(\mathrm{n}=146)$ Registered Nurses $(\mathrm{RN})$ enrolled in the years 2009 and 2011 for a masters award were asked to respond to the question: "Describe the role of a nurse leader in today's healthcare environment". The assignment was voluntary and as an addition to the learning of leadership concepts and techniques which impact organisational outcomes. The RNs were limited to 3,000 words and given seven weeks to complete the assessment task. The scope of the assessment topic was purposefully wide to encourage lateral thinking and an individual perspective of the topic. Of note, all participants were employed in nursing positions as Clinical Nurses $(n=66)$, Level One RNs $(n=56)$, Clinical Nurse Specialists $(n=22)$ and Nurse Practitioners $(n=2)$. These position classifications have as an essential employment criterion a leadership component with Clinical Nurse Specialists and Nurse Practitioners being expected to undertake leadership functions.

\subsection{Ethical considerations}

Ethics approval was obtained from Edith Cowan University's (ECU) Human Research \& Ethics Committee to undertake the study. Participants were informed of the aim, purpose and nature of the study via participant information statement prior to obtaining written consent. The participants were informed that they were able to withdraw consent at any time during the study. Each participant was assigned an identification number to maintain anonymity. The researchers stressed throughout that participation or non-participation in the study did not affect participants overall results in their course.

\subsection{Data collection}

A content analysis was undertaken of all responses submitted. Content analysis involves making the inferences about textual data by systematically and objectively identifying special characteristics or categories and themes within the text. ${ }^{[30]}$ Simply, content analysis is a procedure for organising qualitative data into themes and concepts. The authors 
utilised key terms related to patient safety and leadership identified from the literature for the assessment of each essay. The key terms included were quality, safety, quality improvement and patient risk. Additionally, the authors defined each term to guide the content analysis and to ensure a mutual understanding of the term between the authors. During review of the essays, the authors marked each key term according to the code assigned. The key term was counted for frequency of occurrence in the participants' responses. The content analysis was repeated manually around these terms with the authors moving forwards and backwards in the text to identify related responses.

\section{Definitions}

The following definitions of terms were used to guide the content analysis:

- Quality: "Doing the right things for the right people at the right time and doing them right first time." ${ }^{[31]}$ (p. 16-17).

- Safety: "Perfect harm-free performance and the goal of patient safety is to reduce preventable harm to patients." ${ }^{[32]}$ (p.148).

- Quality improvement: "The degree to which health services for individuals and populations increase the likelihood of desired health outcomes and are consistent with current professional knowledge."[33] (p. 234).

- Patient risk: "The possibility of a loss or other adverse event that has the potential to interfere with a patient's ability to maintain health." ${ }^{[32]}$ (p. 149).

\section{FINDINGS AND DISCUSSION}

Out of 146 responses, 24 nurses acknowledged quality was a leadership function, 19 nurses indicated safety was part of a leadership function, and 32 nurses demonstrated that patient risk management was part of their leadership role. The other responses did not include or use the key terms or phrases indicating these as part of the leadership role. The general category descriptions sought within the essay that the authors believed to be intuitively correlated, that is of leadership and quality and safety were not apparent in the essay text. Theoretic sensitivity, "a loose concept that involves a process of thinking creatively to derive concepts and explanations", [34] (p. 280) led the researchers to the discovery of missing general categories under the intuitive concept grouping amongst RN's understanding of leadership or lack of equating their leadership role with issues of quality and safety.

In this study, when nurses talked about safety, they referred to nurse shortages, and nursing ratios to patients and beds. The overarching response to safety in this instance is more reflective of nursing role protection, through adequate staff

Published by Sciedu Press to patient numbers, rather than in relation to patient care and safety. Here, it may be postulated, the potential oversupply of literature surrounding skill mix, work conditions, bed turnover, and retention and attrition of nurses, has confused and compounded registered nurses understanding of the fundamental requirements of safety leadership. ${ }^{[35-38]}$ What has been missed by this quantitative measure has been the individual nurse's expertise and experience to provide nursing care which is the fundamental unit of measure for skill mix and the main ingredient of safety. Twigg and Duffield ${ }^{[6]}$ note skill mix that is, what each nurse brings to the shift in terms of expertise, experience and education, has a direct influence on patient safety and optimal care.

A possible reason for these results is substantiated by the claim of Richardson and Storrs, ${ }^{[39]}$ where they contend that safety is usually attributed to the higher levels of an organisation that are familiar with, and identify, safety and quality as part of their role. A wide ranging definition of the concept of safety may also confuse the results with grassroots nurses not contemplating the basic tenets of patient safety as within their remit. Nurses see themselves as protective of the patient, able to intervene to prevent specific events where they were involved in immediate feedback, such as doublechecking medications; however, this patient advocacy may not equate to them as patient safety generally. ${ }^{[40]}$ The term 'quality nursing care' was used repeatedly, however there were no words, terms or phrases to provide an example of how this 'quality nursing care' related to safety. The findings of this study indicated that there is room for improvement in the front line nurses knowledge of patient safety as an important tenet of their practice. However, the findings are not supported by research in general, as the majority of evidence indicates that nurses do acknowledge safety as a palpable concern for nursing. ${ }^{[20,41-43]}$

It may be asserted that in the reality of practice, that although nurses are motivated and diligent in patient care, they do not generalise the broader safety leadership messages espoused by management and educators. This is confirmed by our study where patient safety and risk was not specified as a leadership function. Furthermore, there must be a distinction made between safety and quality. Safety requires a cultural shift and a change in the distribution of quality. That is, safety is a responsibility of all individuals involved in healthcare. Kunzel, Kolbe and Grote ${ }^{[23]}$ say that nursing leaders are pivotal in promoting team performance and safety through unambiguous behaviour which is adaptable to situational demands and shared between team members. Quality improvement however, involves incremental improvements in quality and progresses by undertaking rigorous measure of patient outcomes and comparing them overtime. ${ }^{[32]}$ 


\section{RECOMMENDATIONS}

What nurses should understand is that each episode of nursing care is an opportunity for safety and minimising risk and improving outcomes, thus having an effect on overall quality of care. The Australian Office of Safety and Quality in Healthcare 2012 National Safety and Quality Healthcare Standards ${ }^{[44]}$ directly address the individual performance of nurses at the bedside; for example, patient identification, medication administration, clinical handover, and managing pressure areas, are care that nurses do as individuals. The cumulative effect of sound nursing performance ensures the quality of our healthcare system and the protection of the patient.

The crucial concern is the need for educational leadership to espouse safety and quality and continuous improvement to the students as an absolute part of their professional practice and role. ${ }^{[29]}$ It behooves educators and students alike to take a personal 'call to responsibility' for developing a safety vision for the patients in their care. ${ }^{[17,45]}$ All healthcare professions often work within a "perfectibility model"[46] which cause professional division in approaches to organisation safety. ${ }^{[42]}$ What is required is a collaborative and inclusive view of safety to address potential errors in future practice. ${ }^{[47]}$

The content of nursing educational curricula therefore needs to develop and lead the agenda for patient safety through pedagogical or andragogical strategies to achieve this end. Nurse educators who use their leadership skills can transform pa- tient care delivery strategies with improved patient outcomes, reduced mortality and adverse events, and increased patient satisfaction; however, the mental model of the nurse needs to embrace safety as central to the practice of leadership.

\section{Conclusion}

This discussion paper offers insight into the postgraduate nursing student's view on the leadership role in today's healthcare environment. Few responses tied nurse leader responsibilities to patient safety and quality. Therefore, it is thought that the students lack the intuition of linking leadership and patient safety at this stage of professional and educational development. According to Benner, ${ }^{[9]}$ an intuitive thinker develops through learning, experience and evaluation of performance. Those nurses working in managerial or leadership roles have the responsibility of patient safety and involvement with quality activities, thus have a greater understanding of the nurse leader role and safety. However, there is a need for front line nurses to understand the importance of patient safety given the detrimental consequences. The importance of safety leadership cannot be overemphasised and is the responsibility of all nurses no matter what level of experience to ensure patient safety, improve quality and minimise risk.

\section{CONFLicts OF InTEREST Disclosure}

The authors declare that there is no conflict of interest statement.

\section{REFERENCES}

[1] World Health Organisation, WHO Patient Safety. WHO Patient Safety Research: Better Knowledge for Better Care. Geneva: WHO Publication. 2009.

[2] Kho ME, Carbone JM, Lucas J, et al. Safety climate survey: reliability of results from a multi-centre ICU survey. Quality and Safety in Healthcare. 2005; 14: 273-278. http://dx.doi.org/10.1136/q shc. 2005.014316

[3] Hutchinson A, Cooper KL, Dean JE, et al. Use of a safety climate questionnaire in UK healthcare: factor structure reliability and usability. Quality and Safety in Healthcare. 2006; 15: 347-353. http://dx.doi.org/10.1136/qshc.2005.016584

[4] Hendricks JM, Cope VC. Generational diversity: What nurse managers need to know. Advanced Journal of Nursing. 2012; 69(3): 717 725. http://dx.doi.org/10.1111/j.1365\T1\textendash6 48.2012.06079. $\mathrm{x}$

[5] Tourangeau AE, Cranley LA, Jeffs L. Impact of nursing on hospital patient mortality: a focused review and related policy implications. Quality and Safety in Health Care. 2006; 15: 4-8. http: //dx.doi.org/10.1136/qshc.2005.014514

[6] Twigg D, Duffield C. A review of workload measures: a context for a new staffing methodology in Western Australia. International Journal of Nursing Studies. 2009; 46(1): 132-140. http: //dx.doi.org/10.1016/j.ijnurstu.2008.08.005
[7] Aiken L, Sermmeus W, KoenVandenHeede H, et al. Patient safety, satisfaction, and quality of hospital care: cross sectional surveys of nurses and patients in 12 countries in Europe and the United States. British Medical Journal. 2012; 344: e1717. http://dx.doi.org /10.1136/bmj.e1717

[8] Burston S, Chaboyer W, Wallis M, et al. A discussion of approaches to transforming care: contemporary strategies to improve patient safety. Journal of Advanced Nursing. 2011; 67(11): 2488-2495. http://dx.doi.org/10.1111/j.1365-2648.2011.05706.x

[9] Benner P. From novice to expert: Excellence and power in clinical nursing practice. Menlo Park, CA: Addison-Wesley; 1984.

[10] Institute of Medicine. Keeping Patients Safe: Transforming the Work Environment of Nurses. Washington, DC: National Academy of Sciences. 2003.

[11] Cook AF, Hoas H, Guttmannova KMA, et al. An error by any other name. American Journal of Nursing. 2004; 104(6): 32-43. http://dx.doi.org/10.1097/00000446-200406000-00025

[12] Australian Nursing and Midwifery Competencies [ANMC]. Australian Nursing and Midwifery Competency Standards for Registered Nurses. Canberra: Australian Nursing and Midwifery Council. 2006

[13] Kennedy I. Learning from Bristol: The Report of the Public Inquiry into Children's Heart Surgery at the Bristol Royal Infirmary 19841995. London: The Stationary Office. 2001.

[14] Sinclair CM. The Report of the Manitoba Cardiac Inquest: An Inquiry into Twelve Deaths at the Winnipeg Health Sciences Centre in 
1994 [Internet]. Winnipeg Provincial Court of Manitoba, 2000 [cited 2013 June 1]. Available from: http://www.pediatriccardiac inquestamb.ca

[15] Douglas N, Fahy K, Robinson J. Report of the Inquiry into Obstetric and Gynaecological Services at King Edward Memorial Hospital 1990-2000 [Internet]. Perth, Western Australia: Department of Health, 2001 [cited 2013 June 9]. Available from: http://www.kemh.health.wa.gov.au/general/KEMH _Inquiry/reports.htm

[16] Gregory DM, Guse LW, Dick DD, et al. Patient safety: Where is nursing education? Journal of Nursing Education. 2007; 46(2): 79-82. PMid:17315567

[17] Chernot TM, Daniel LG. Frameworks for patient safety in the nursing curricula. Journal of Nursing Education. 2010; 49(10): 559-568. http://dx.doi.org/10.3928/01484834-20100730-02

[18] Travaglia JF, Debono D, Spigleman AD, et al. Clinical governance: a review of key concepts in the literature. Clinical Governance: An International Journal. 2011; 16(1): 62-77. http://dx.doi.org/1 $0.1108 / 14777271111104592$

[19] Squires M, Tourangeau A, Spence LH, et al. The link between leadership and safety outcomes in hospitals. Journal of Nursing Management. 2010; 18(8): 914-925. http://dx.doi.org/10.1111/j.1 365-2834.2010.01181.x

[20] McNamara S. The future of nursing and patient safety: The nurse's role. AORN Journal. 2011; 93(5): 614-618. http://dx.doi.org /10.1016/j.aorn.2011.01.010

[21] Higgs M, Rowland D. Emperors with clothes on: The role of selfawareness in developing effective change leadership. Journal of Change Management. 2010; 10(4): 369-385. http://dx.doi.o rg/10.1080/14697017.2010.516483

[22] Wong CA, Spence LH, Cummings GG. Authentic leadership and nurses' voice behaviour and perceptions of care quality. Journal of Nursing Management. 2010; 18: 889-900. http://dx.doi.org/1 $0.1111 / \mathrm{j} .1365-2834.2010 .01113 . x$

[23] Kunzel B, Kolbe M, Grote G. Ensuring patient safety through effective leadership behaviour: A literature review. Safety Science. 2010; 48: 1-17. http://dx.doi.org/doi:10.1016/j.ssci.2 009.06 .004

[24] Steven A, Magnusson C, Smith P, et al. Patient safety in nurse education: contexts, tensions and feeling safe to learn. Nurse Education Today. 2014; 34(2): 277-284. PMid:23726756 http://dx.doi .o $\mathrm{rg} / 10.16 / \mathrm{j} . \mathrm{nedt} .2013 .04 .025$

[25] Alltree M, Cooke H, Wakefield A. Patient safety in an English preregistration nursing curriculum. Nurse Education in Practice. 2008; 8(4): 239-248. PMid:17945535 http://dx.doi.org/10.1016/j .nepr.2007.09.003

[26] United Kingdom Nursing and Midwifery Council. The Code: Standards of Conduct, Performance and Ethics for Nurses and Midwives. London: Nursing and Midwifery Council. 2008.

[27] American Nurses Association. Code of Ethics with Interpretive Statements. Washington, USA: American Nurses Association. 2001.

[28] Wakefield A, Attree M, Braidman I, et al. Patient safety: do nursing and the medical curricula address this theme? Nurse Education Today. 2005; 25: 333-340. http://dx.doi .org/10.1016/j.nedt. 2005.02 .004

[29] Vaismoradi M, Salsali M, Marck P. Patient safety: nursing students' perspectives and the role of nursing education to provide safe care. International Nursing Review. 2011; 58: 434-442. http: //dx.doi.org/10.1111/j.1466-7657.2011.00882.x
[30] Gray DE. Doing research in the real world (2nd ed.). Los Angeles: Sage; 2009.

[31] Wright J, Hill P. Clinical Governance. Edinburgh: Churchill Livingstone; 2003.

[32] Warburton R. Quality and patient safety: how do we get there from her? In R. Sorensen \& R. Iedema (Eds.), Managing clinical processes in health services. Sydney: Churchill Livingstone. 2008.

[33] Daly J, Speedy S, Jackson D. Nursing Leadership. Sydney: Churchill Livingstone. 2007.

[34] Reinard JC. Introduction to communication research (4th ed.). New York: McGraw-Hill. 2007.

[35] Hill KS. Improving quality and patient safety by retaining nursing expertise. OJIN: The Online Journal of Issues in Nursing [Internet]. 2010 [cited 2013 June 9]; 15(3). Available from: http: //www . medscape.com/viewarticle/729397

[36] Hines PA, Yu KM. The changing reimbursement landscape: Nurses' role in quality and operational excellence. Nursing Economics. 2009; 27(1): 7-14. PMid:19331307

[37] Laschinger HKS, Leiter MP. The impact of nursing work environments on patient safety outcomes. The Journal of Nursing Administration. 2006; 36(5): 259-267. http://dx.doi.org/10.1097/0 0005110-200605000-00019

[38] Swearingen S. A journey to leadership: Designing a nursing leadership development programme. The Journal of Continuing Education in Nursing. 2009; 40(3): 107-112. http://dx.doi.org/10.3928 /00220124-20090301-02

[39] Richardson A, Storr J. Patient safety: a literature review on the impact of nursing empowerment, leadership and collaboration. International Nursing Review. 2010; 57: 12-21. PMid:20487470 http://dx.doi.org/10.1111/j.1466-7657.2009.00757.x

[40] Elder NC, Brungs SM, Nagy M, et al. Intensive care unit nurses' perceptions of safety after a highly specific safety intervention. Quality and Safety in Health Care. 2008; 17: 25-30. PMid:18245216 http://dx.doi.org/10.1136/qshc.2006.021949

[41] Feng X, Bobay K, Weiss M. Patient safety culture in nursing: a dimensional concept analysis. Journal of Advanced Nursing. 2008; 63(3): 310-319. http://dx.doi.org/10.1111/j.1365-2648. 2008.04728. $\mathrm{x}$

[42] Hellings J, Schrooten W, Klazing NS, et al. Improving patient safety culture. International Journal of Health Care Quality Assurance. 2010; 23(5): 489-506. http://dx.doi.org/10.1108/09526861011050529

[43] Thompson PA, Navarra MB, Antonson N. Patient safety: The four domains of nursing. Nursing Economics. 2005; 23(6): 331-333. PMid: 16459908

[44] Government of Western Australia. National and State Update, Issue 2 November. Perth, Western Australia: Office of Safety and Quality in Healthcare. 2012.

[45] The National Quality Forum. National Quality Forum Safe Practices for Better Healthcare 2009 Update [Internet]. Washington, DC: The National Quality Forum, 2009 [cited 2013 January 22]. Available from: http://www.qualityforum.org/Publications/2009 /03/Safe_Practices_for_Better_Healthcare $\%$ E $2 \% 80 \% 932$ 009_Update.aspx

[46] Leap L. Error in medicine. Journal of American Medical Association 1994; 272: 1851-1857. http://dx.doi.org/10.1001/jama.19 94.03520230061039

[47] Vogelsmeier A, Scott-Cawiezell J, Miller B. Influencing leadership perceptions of patient safety through just culture training. Journal of Nursing Care Quality. 2010; 25(4): 288-294. PMid:20220531 http://dx.doi.org/10.1097/NCQ. Ob013e3181d8e0f2 